\title{
Aldehyde dehydrogenase 2 inhibits inflammatory response and regulates atherosclerotic plaque
}

\author{
Chang Pan ${ }^{1,2,3,4, *}$, Jun-hui Xing ${ }^{1,2,3,4, *}$, Cheng Zhang ${ }^{2,5, *}$, Ying-mei Zhang ${ }^{6, *}$, Lue- \\ tao Zhang ${ }^{1,2,3,4, *}$, Shu-jian Wei ${ }^{1,2,3,4}$, Ming-xiang Zhang ${ }^{2}$, Xu-ping Wang ${ }^{2}$, Qiu-huan \\ Yuan ${ }^{1,2,3,4}$, Li Xue ${ }^{1,2,3,4}$, Jia-li Wang ${ }^{1,2,3,4}$, Zhao-qiang Cui ${ }^{2}$, Yun Zhang ${ }^{2,5}$, Feng Xu1,2,3,4 \\ and Yu-guo Chen $1,2,3,4$ \\ ${ }^{1}$ Department of Emergency and Chest Pain Center, Qilu Hospital, Shandong University, Ji'nan, China \\ ${ }^{2}$ Key Laboratory of Cardiovascular Remodeling and Function Research, Ministry of Education and Ministry of Public Health of \\ People's Republic of China, Qilu Hospital, Shandong University, Ji'nan, China \\ ${ }^{3}$ Institute of Emergency and Critical Care Medicine, Qilu Hospital, Shandong University, Ji'nan, China \\ ${ }^{4}$ The Key Laboratory of Emergency and Critical Care Medicine affiliated to Health Commission of Shandong Province, Qilu \\ Hospital, Shandong University, Ji'nan, China \\ ${ }^{5}$ Department of Cardiology, Qilu Hospital, Shandong University, Ji'nan, China \\ ${ }^{6}$ Shanghai Institute of Cardiovascular Diseases, Zhongshan Hospital, Fudan University, Shanghai, China \\ * These authors have contributed equally to this work \\ Correspondence to: Feng Xu, email: xufengsdu@126.com \\ Yu-guo Chen, email: chen919085@126.com
}

Keywords: ALDH2, polymorphism, atherosclerotic plaque vulnerability, inflammation, MAPK signaling pathway, Pathology Section Received: April 22, 2016

Published: May 15, 2016

\section{ABSTRACT}

Previous studies demonstrated that aldehyde dehydrogenase 2 (ALDH2) rs671 polymorphism, which eliminates ALDH2 activity down to $1 \%-6 \%$, is a susceptibility gene for coronary disease. Here we investigated the underlying mechanisms based on our prior clinical and experimental studies. Male apoE-/- mice were transfected with GFP, ALDH2-overexpression and ALDH2-RNAi lentivirus respectively $(n=20$ each) after constrictive collars were placed around the right common carotid arteries. Consequently, ALDH2 gene silencing led to an increased en face plaque area, more unstable plaque with heavier accumulation of lipids, more macrophages, less smooth muscle cells and collagen, which were associated with aggravated inflammation. However, ALDH2 overexpression displayed opposing effects. We also found that ALDH2 activity decreased in atherosclerotic plaques of human and aged apoE-/- mice. Moreover, in vitro experiments with human umbilical vein endothelial cells further illustrated that, inhibition of ALDH2 activity resulted in elevating inflammatory molecules, an increase of nuclear translocation of NF-KB, and enhanced phosphorylation of NF-KB p65, AP-1 c-Jun, Jun-N terminal kinase and p38 MAPK, while ALDH 2 activation could trigger contrary effects. These findings suggested that ALDH2 can influence plaque development and vulnerability, and inflammation via MAPK, NFKB and AP-1 signaling pathways. 


\section{INTRODUCTION}

Acute coronary syndrome (ACS) is the main clinical subtype of atherosclerotic coronary heart disease (CAD) with an increasing morbidity and mortality. It is well known that aldehyde dehydrogenase 2 (ALDH2), as the key enzyme for classical alcohol metabolism, displays an important functional single-nucleotide polymorphism (SNP), or rs671-Glu504Lys polymorphism [1]. Previous studies demonstrated that ALDH2 rs671 mutant displays significantly reduced enzyme activity (1\%-6\% of wildtype) $[2,3]$. This mutation exists in $30 \%-50 \%$ of East Asians, which is $\sim 6 \%$ of the world population [1]. The allele occurs in most regions of China, Japan, Korea, Mongolia, and Ecuador with the highest frequency found in Indians of Ecuador Highlands and Southeast Asia. The allele is also present in certain American populations such as Native and Indian Americans although it is rare in Caucasians [4]. Previous studies have shown that the ALDH2 Glu504Lys polymorphism is associated with some drinking-related diseases such as alcoholic cardiomyopathy, alcohol liver cirrhosis and alcohol chronic pancreatitis $[5,6]$. Recently, some studies have indicated that ALDH2 and its polymorphisms are involved in ACS and CAD. Most of these work of ALDH2 tended to focus on myocardium, with limited information on the vasculature. Several surveys, including studies conducted in Japan, China and Korea, have indicated that the ALDH2 Glu504Lys polymorphism is closely associated with myocardial infarction and/or ACS in East Asians and the ALDH2 mutant genotypes $(* 1 / * 2$ and $* 2 / * 2)$ are considered independent risk factors of ACS [7-9]. In 2012, a genome-wide association study of common SNPs identified that ALDH2 is a CAD susceptibility gene [10]. However, the exact molecular mechanisms underlying how ALDH2 affects the occurrence of ACS or CAD remain unclear.

One earlier study from our group demonstrated that ALDH2 mutation is associated with decreased high-density lipoprotein cholesterol (HDL-C) levels [11], suggesting that ALDH2 might influence atherosclerosis progression and subsequently $\mathrm{CAD}$ via lipid accumulation. Our data found that ox-LDL may decrease ALDH2 dehydrogenase activity obviously, although inhibition of Poly(ADP-ribose) polymerase restores the ALDH2 dehydrogenase activity by preventing expression of SIRT3 and maintaining the acetylation level of ALDH2 [12]. Moreover, one of our studies displayed that ALDH2 activation with hyperacetylation by SIRT3 inactivation improves human aortic endothelial function [13]. Furthermore, we also found that ALDH2 inhibition by hyperglycemia aggravates mitochondrial impairment, which is essential to cellular energy metabolism [14]. Recently, our studies further indicated that the ALDH2 mutation is tied with levels of high-sensitivity C-reactive protein, a classical inflammatory biomarker, and the number of circulating endothelial progenitor cells [15]. In addition, previous studies showed that overexpression of ALDH2 attenuates myocardial apoptosis [16] and ALDH2 plays an important role in myocardial protection [17-19]. It is well conceived that lipids, endothelial dysfunction or injury-repair imbalance, and inflammation play critical roles in atherosclerosis progression and atherosclerotic plaque vulnerability, which are recognized as a pivotal mechanism of ACS [20]. Furthermore, inflammation, which can be triggered by oxidative stress, is the main cause of many human diseases, including cardiovascular diseases [21]. Therefore, we hypothesized that ALDH2 might play a role in vasculature by the way of influencing plaque development, vulnerability and inflammation, leading to acute coronary events. This hypothesis is further supported by recent reports which indicated that ALDH2 with different activities has different impacts on oxidative stress and subsequent inflammation. Recent studies found that functional ALDH2 could metabolize aldehydes, such as the endogenous oxidative stressor 4-hydroxy-2-nonenal (4-HNE), into much less reactive chemical species [22]. Our recent study also revealed that improvement in ALDH2 activity is capable of ameliorating oxidative stress [23].

In the present study, to investigate the effect of ALDH2 on vasculature, we hypothesized that ALDH2 could affect plaque development, stability and inflammation. To validate this hypothesis and investigate the underlying signaling pathways, a series of in vivo and in vitro experiments were designed and performed.

\section{RESULTS}

\section{ALDH2 expression and activity in mice}

All mice were healthy in the process of collar placement and lentivirus treatment (Figure 1A), but one mouse in the Lv-ALDH2-RNAi group and one mouse in the Lv-ALDH2-overexpression group were lost before the day of sacrifice. To estimate the efficiency of lentivirusmediated gene overexpression and knockdown in vivo, the levels of ALDH2 protein expression in carotid plaques and ALDH2 activity of aortas were detected (Figure 1B1D). Compared with the Lv-green fluorescent protein (GFP) group, ALDH2 protein expression levels in the Lv-ALDH2-overexpression group and Lv-ALDH2RNAi group were increased by approximately $150 \%$ and decreased by $80 \%$, respectively (both $\mathrm{P}<0.05$, Figure 1B and $1 \mathrm{C}$ ). Moreover, in accordance with protein expression levels in different groups, the activity of ALDH2 in the Lv-ALDH2-overexpression group was increased but decreased in the Lv-ALDH2-RNAi group (both $\mathrm{P}<0.05$, Figure 1D). 
A
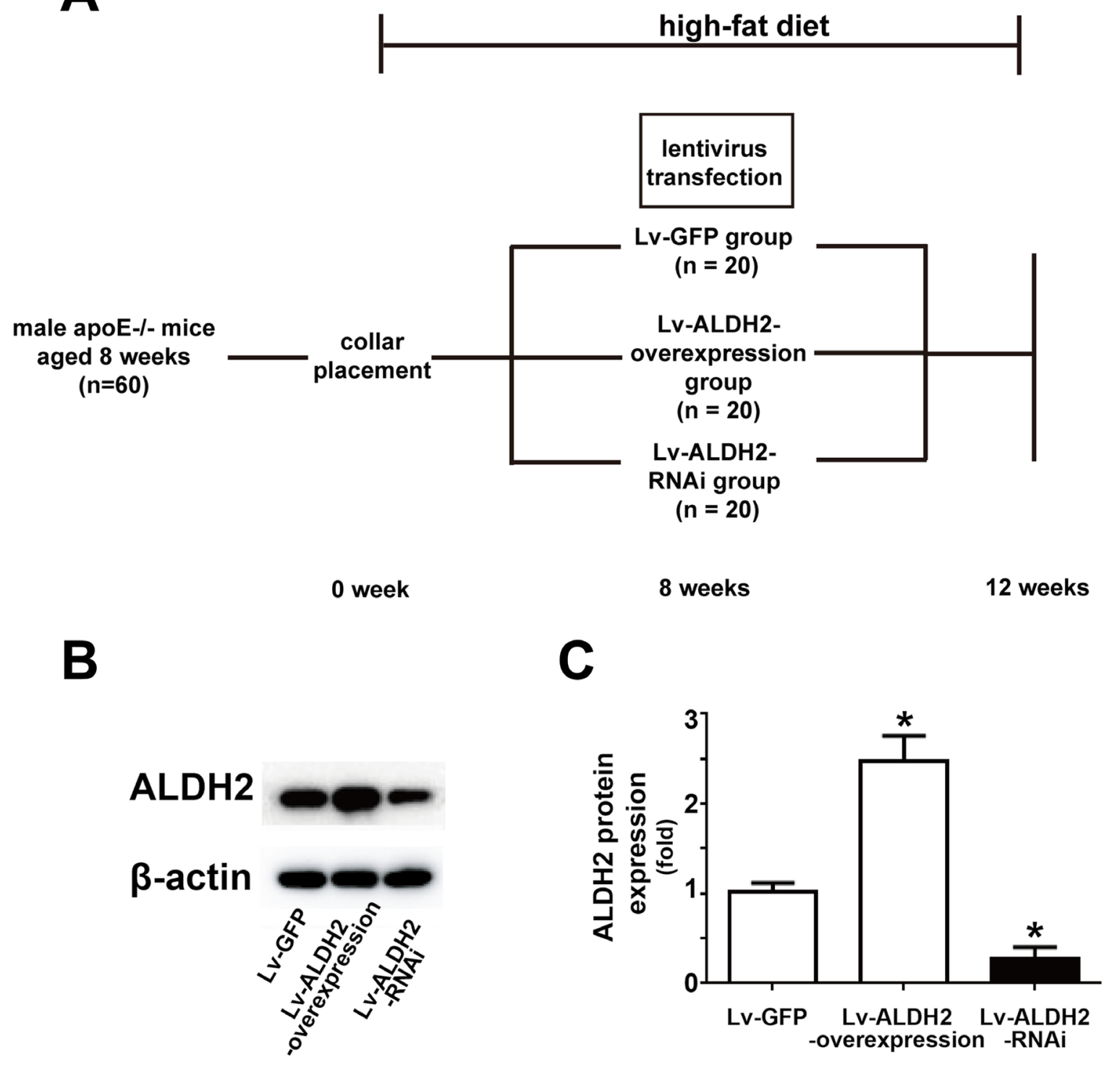

D

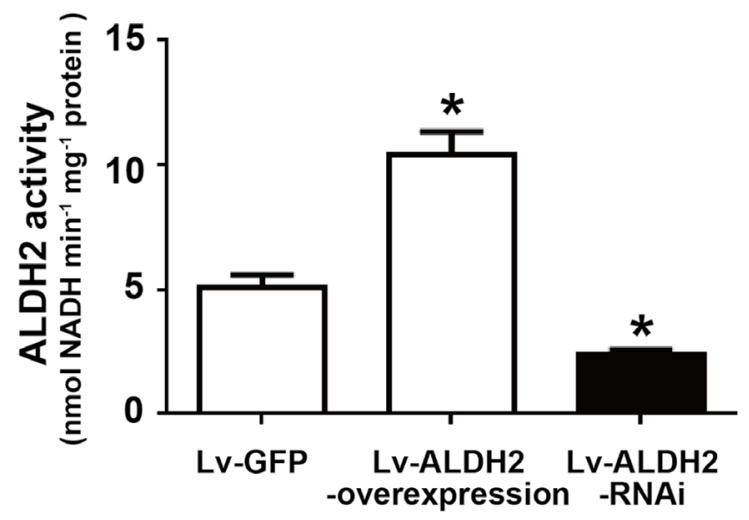

Figure 1: Time line, grouping and ALDH2 expression after lentivirus transfection in apoE-/- mice. A. Time line and grouping of the mice experiment. B. Representative blot of ALDH2 protein expression in three groups of the apoE-/- mice. C. Statistical analysis of relative ALDH2 expression in three groups. D. Histograms of ALDH2 activity in three groups. All data were showed as mean \pm SEM. *: $P<0.05$ vs Lv-GFP group. 


\section{Effect of ALDH2 on body weight and serum lipid levels}

For each group of mice, body weight and the serum concentrations of lipid were measured at the end of the experiment. Neither the carotid collar placement nor the lentivirus injection had effects on their body weight (Table S1). There were also no significant differences of serum levels of total cholesterol, triglyceride, low-density lipoprotein cholesterol (LDL-C) and HDL-C among the
A
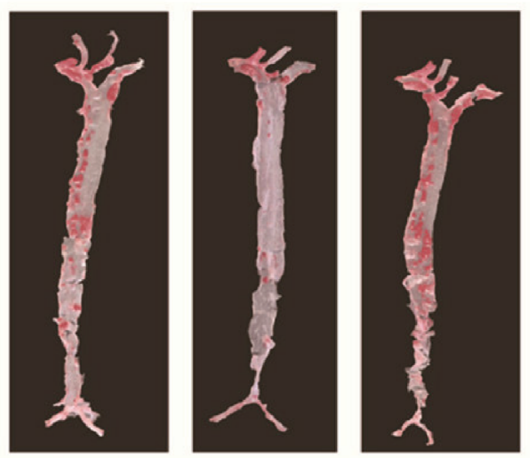

B

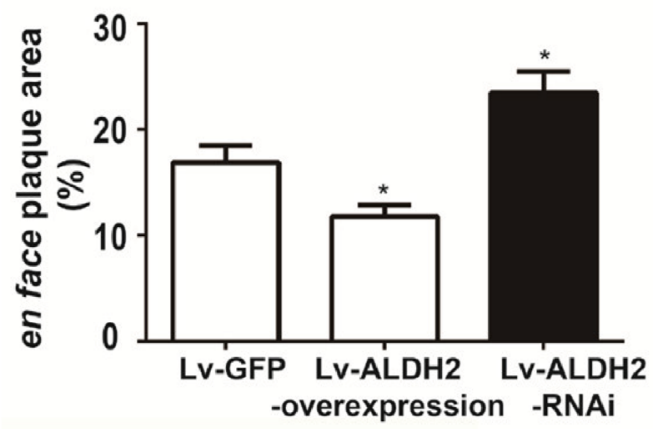

C

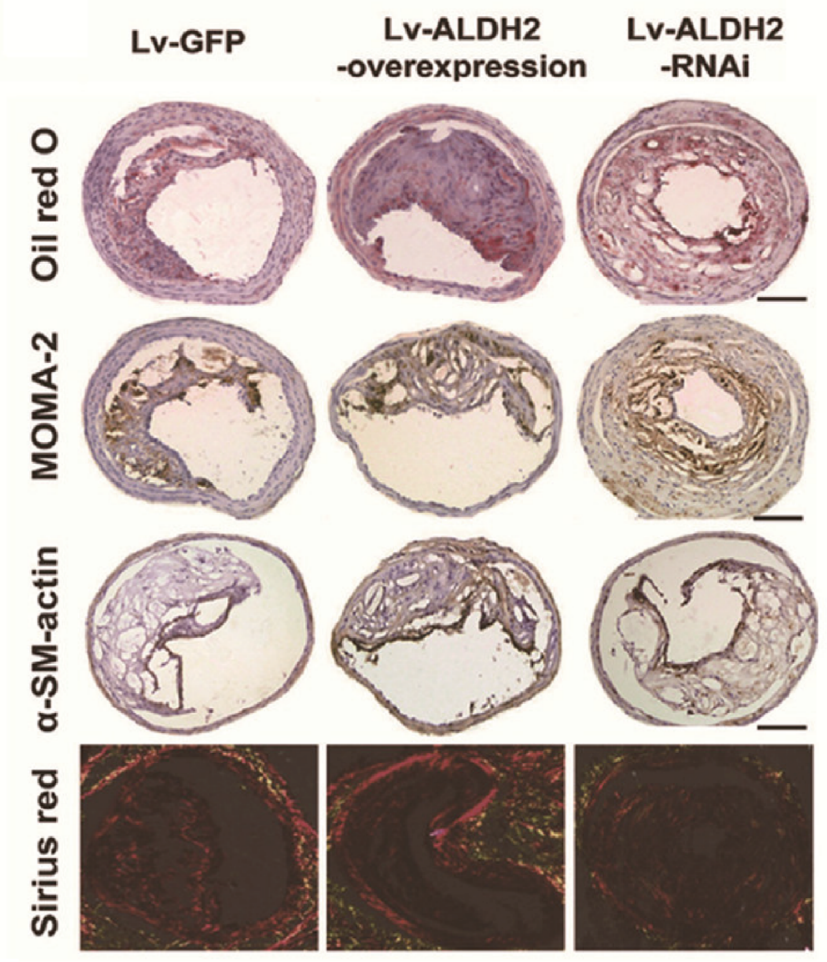

D

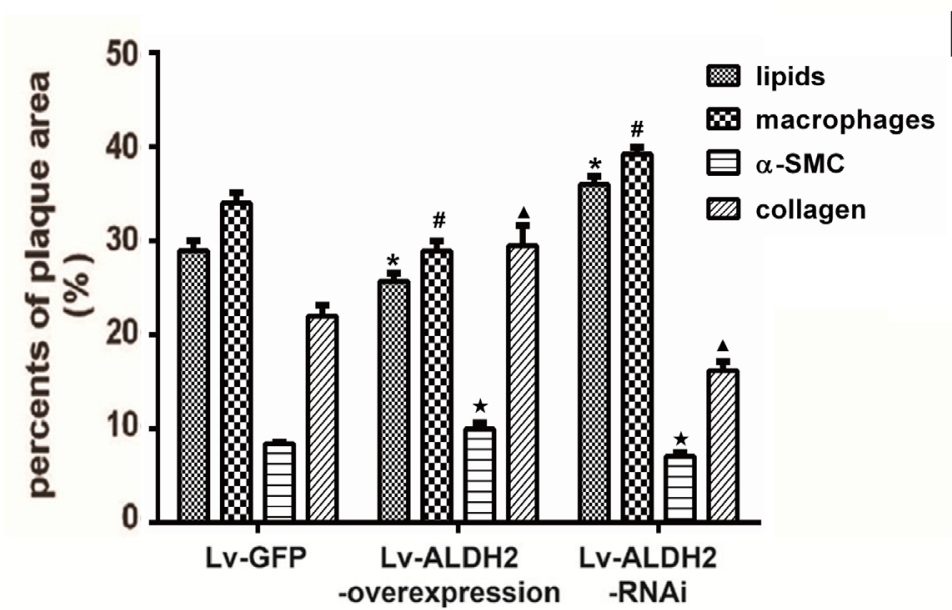

Figure 2: Changes of atherosclerotic plaques in aortas and carotid plaques composition in apoE-/- mice after lentivirus transfection. A. Representative en face photographs of aortas in three groups showing oil red O-stained atherosclerotic plaques. B. Quantitative measurement of en face plaque area (\%) in the whole aorta. *: $P<0.05$ v Lv-GFP group. C. Representative Oil-red O staining, MOMA-2 immunostaining, $\alpha$-SM-actin immunostaining and Sirius-red staining in three groups of the apoE-/- mice; the bars represent $100 \mu \mathrm{m}$. D. Column graph of quantification of staining results in Figure $2 \mathrm{C}$ by Image-Pro Plus 6.0 Software. ${ }^{*},{ }^{*},{ }^{\star},{ }^{\mathbf{\Delta}}: P<0.05$, percent of lipids, macrophages, $\alpha$-SMCs and collagen compared with Lv-GFP group, respectively. All data were presented as mean \pm SEM. E. Bar chart of vulnerable index in three groups of the apoE-/- mice. The vulnerability indexs in the Lv-GFP, Lv-ALDH2-overexpression and LvALDH2-RNAi groups are $2.10 \pm 0.10,1.40 \pm 0.10$ and $3.20 \pm 0.19$, respectively. *: $P<0.05 v s$ Lv-GFP group. 
Lv-ALDH2-overexpression group, the Lv-ALDH2-RNAi group and the Lv-GFP group (Table S2), suggesting that ALDH2 gene transfer has little effects on serum lipid levels.

\section{Effect of ALDH2 on the extent of aortic atherosclerosis}

To evaluate the effect of ALDH2 on the extent of aortic atherosclerosis, total lesion area of aortas were measured using oil-red $\mathrm{O}$ staining at 4 weeks after lentivirus transfection. The extent of atherosclerosis in Lv-GFP group was intermediate between that observed in the Lv-ALDH2-overexpression and Lv-ALDH2RNAi groups. Alternatively, Lv-ALDH2-overexpression group showed significantly reduced en face plaque area compared with the Lv-GFP group $(11.80 \% \pm 1.10 \%$ vs $16.92 \% \pm 1.55 \%, \mathrm{P}<0.05$ ), while the Lv-ALDH2RNAi group had increased atherosclerotic lesion areas $(23.51 \% \pm 1.99 \%, \mathrm{P}<0.05$, Figures $2 \mathrm{~A}$ and $2 \mathrm{~B})$.

\section{Effect of ALDH2 on component changes of carotid plaques}

The relative content of lipids, macrophages, $\alpha$-smooth muscle cells (SMCs) and collagen in the plaques was measured by histological and immunohistochemical staining (Figure 2C). In Lv-ALDH2-overexpression group, the ratios of lipids and macrophages were lower in carotid plaques, whereas $\alpha$-SMCs and collagen ratios were higher compared with the Lv-GFP group $(\mathrm{P}<0.05)$. However, plaques in the Lv-ALDH2-RNAi group displayed a higher content of lipids and macrophages, but a lower content of $\alpha$-SMCs and collagen ( $\mathrm{P}<0.05$, Figures $2 \mathrm{C}$ and $2 \mathrm{D})$. These led to a change of the plaque vulnerability index. Compared with the Lv-GFP group, the vulnerability index in the Lv-ALDH2-overexpression group is lower, but the vulnerability index in the Lv-ALDH2-RNAi group is higher $(\mathrm{P}<0.05$, Figure 2E).

\section{Effect of ALDH2 on the expression of inflammation markers in carotid plaques}

The protein expression levels of intercellular adhesion molecule-1 (ICAM-1), matrix metalloproteinase-2 (MMP-2), interleukin-6 (IL-6) and monocyte chemotactic protein-1 (MCP-1) were detected by western blot (Figure 3). Compared with the Lv-GFP group, the expression of ICAM-1, MMP-2, IL-6 and MCP-1 were reduced approximately by $70 \%, 50 \%, 80 \%$ and $50 \%$ in the Lv-ALDH2-overexpression group but increased approximately by $100 \%, 90 \%, 300 \%$ and $100 \%$ in the Lv-ALDH2-RNAi group $(\mathrm{P}<0.05)$, respectively.

\section{ALDH2 expression and activity in atherosclerotic plaques}

We further detected ALDH2 expression and activity in postmortem human coronary arteries. Compared with human coronary arteries without plaques, both ALDH2 expression and activity in the atherosclerotic vessels were decreased (both $\mathrm{P}<0.05$ ) (Figure 4A-4C). Similarly,

\section{A}

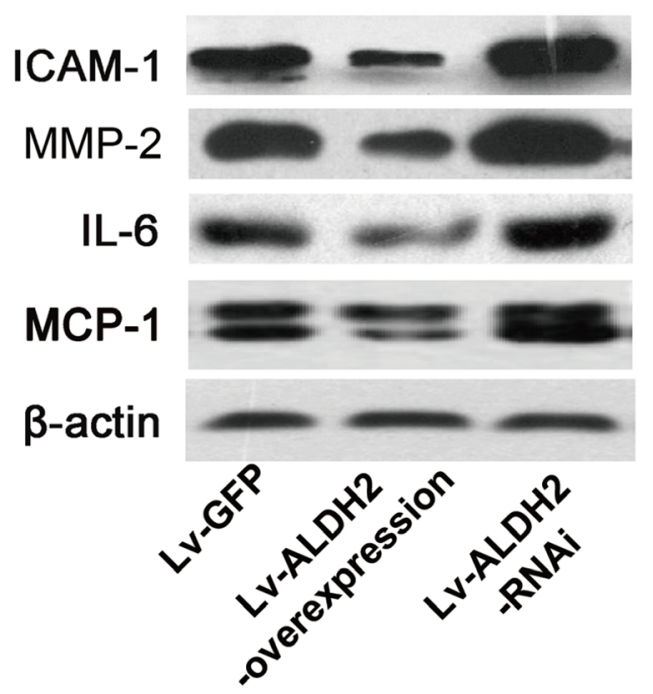

B

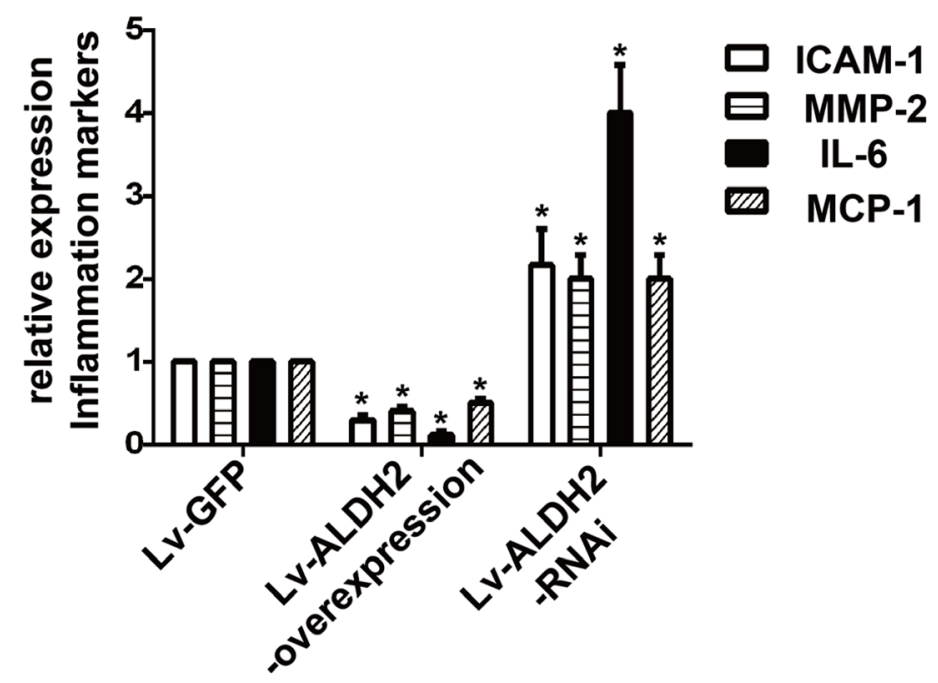

Figure 3: Protein expression of inflammatory cytokines in three groups of the apoE-/- mice after lentivirus transfection. A. Representative western images of ICAM-1, IL-6, MMP-2 and MCP-1 in three groups. B. Statistical analysis of the data presented in Figure $3 \mathrm{~A}$. The data were presented as mean \pm SEM. *: $P<0.05$ vs Lv-GFP group. 
ALDH2 activity in the aged apoE-/- mouse aortas with plaques was also lower than that in normal aortas (Figure 4D). In addition, we found that ALDH2 was expressed in endothelial cells and SMCs in human coronary arteries (Figure 4E).

\section{Time- and dose-dependent effects of LPS on expression of MCP-1 and ICAM-1 protein in HUVECs}

To select the optimal concentration and incubation time for lipopolysaccharide (LPS), expression of MCP-1 and ICAM-1 protein was measured in human umbilical vein endothelial cells (HUVECs) exposed to LPS at various concentrations for 12 hours and 100ng/ml LPS for various times. High concentrations and long LPS incubation times resulted in significant MCP-1 and ICAM1 production (Figure S1). Thus, we chose 100ng/ml LPS and a 12-hour HUVEC incubation time.

\section{Effects of ALDH2 on the expression of MCP-1 and ICAM-1 protein in HUVECs activated with LPS}

We first used daidzin, a known potent and selective inhibitor of ALDH2 [24], to inhibit ALDH2 activity. Daidzin $(25 \mu \mathrm{M})$ reduced ALDH2 activity by approximately $50 \%$ with little effect from the solvent DMSO (Figure S2A). As shown in Figure 5A, both MCP1 and ICAM-1 protein were increased after inhibiting ALDH2 activity. To determine the exact effect of ALDH2, we used Alda-1, a novel small molecule which can activate catalysis for both wild-type ALDH2 (ALDH2*1) and $\mathrm{ALDH} 2 * 2[25,26]$, to stimulate ALDH2 activity. We demonstrated that $20 \mu \mathrm{M}$ Alda-1 increased ALDH2 activity approximately by $80 \%$ with no effect from the solvent DMSO (Figure S2B). As shown in Figure 5B, Alda-1 significantly inhibited the expression of MCP-1 and ICAM-1 proteins.

\section{Effects of ALDH2 on the expression of NF-KB and AP-1 transcription factors}

Nuclear factor $-\kappa \mathrm{B}(\mathrm{NF}-\kappa \mathrm{B})$ is a pivotal regulator of inflammation, and activation of $\mathrm{NF}-\mathrm{kB}$ is required to induce expression of MCP-1 and ICAM-1 in LPSactivated endothelial cells [27]. The location of NF- $\kappa \mathrm{B}$ subunit P65 was determined using western blot. LPS at $100 \mathrm{ng} / \mathrm{ml}$ triggered $\mathrm{NF}-\kappa \mathrm{B}$ p65 subunit translocation from the cytoplasm to the nucleus. HUVECs pretreated with daidzin displayed an increase of nuclear translocation (Figure 5C), and Alda-1 inhibited the nuclear translocation (Figure 5E). In addition, the phosphorylation of NF$\kappa \mathrm{B}$ p $65^{\mathrm{S} 536}$, as a type of posttranslational modification, is reported to contribute to the activity of NF- $\kappa \mathrm{B}$ p65 and prolong the activity of NF- $\mathrm{BB}$ in the nucleus [28]. As shown in Figure 5D, the phosphorylation of NF- $\kappa \mathrm{B}$ p65 $5^{5536}$ was increased in LPS-treated HUVECs pretreated with daidzin compared with that in HUVECs treated with LPS alone, whereas it was decreased in Alda-1-treated HUVECs (Figure 5F). Increasing evidence indicates the importance of transcription factor activator protein-1 (AP-1) independent of NF- $\mathrm{BB}$ in the pathogeneses of endothelial inflammation. The phosphorylation of AP-1 c-Jun ${ }^{\text {ser73 }}$ could enhance its transactivation potential. In Figure 5D, the phosphorylation of AP-1 c-Jun ${ }^{\mathrm{S} 73}$ was enhanced in LPS-treated HUVECs pretreated with daidzin

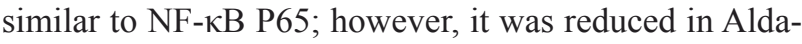
1-treated HUVECs (Figure 5F). In addition, ALDH2 had little effect on the expression of AP-1 c-Jun protein.

\section{Effects of ALDH2 on the expression of the MAPK system}

Mitogen-activated protein kinase (MAPK) cascade, which can be upstream of NF- $\mathrm{kB}$ and AP-1, accounts for the cellular injury mechanisms during various stresses, including inflammation response. Next, we analyzed whether such a cascade was responsible for the regulation of MCP-1 and ICAM-1 expression by ALDH2. Western blot results revealed that phosphorylation of Jun-N terminal kinase (JNK) and p38 MAPK but not extracellular signal-regulated kinase 1/2 (ERK1/2) were further enhanced in LPS-activated HUVECs pretreated with daidzin compared with that in HUVECs treated with LPS alone (Figure 6A). Moreover, we confirmed that

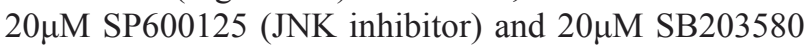
(p38 MAPK inhibitor) preferentially inhibited expression of MCP-1 and ICAM-1 protein in LPS-activated HUVECs pretreated with daidzin compared with HUVECs treated with LPS alone (Figure 6B). As shown in Figure 6C, Alda1 inhibited phosphorylation of JNK and p38 MAPK but not ERK1/2.

\section{DISCUSSION}

The major findings of our present study were that ALDH2 overexpression in the vulnerable carotid plaque of apoE-/- mice led to higher ALDH2 activity and a more stable plaque with less accumulation of lipids, fewer macrophages, more SMCs and collagen, as well as with reduced inflammatory factors. However, gene silencing of ALDH2 displayed opposing effects. In vitro experiments demonstrated that ALDH2 influenced inflammatory molecules including MCP-1 and ICAM-1 through some members of the MAPK family (JNK and p38 MAPK, but not ERK1/2) and the NF- $\mathrm{KB}$ and AP-1 signaling pathways. To the best of our knowledge, this is the first report that ALDH2 can directly influence plaque development, 
A

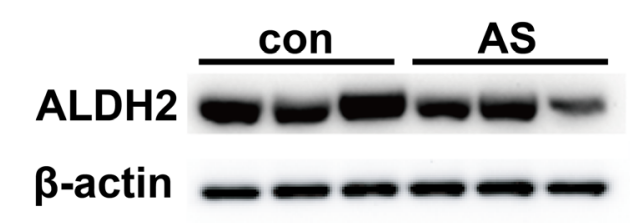

C

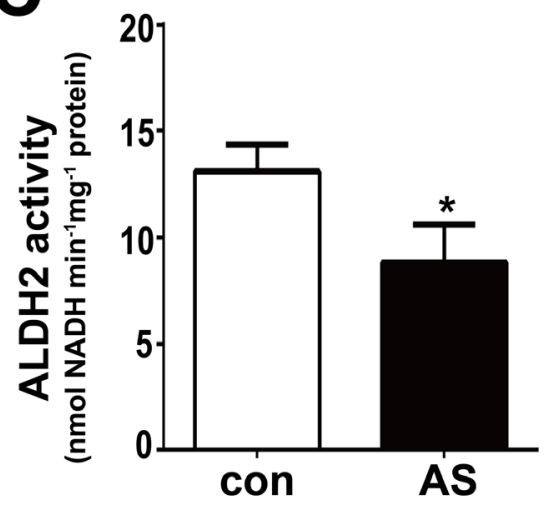

B

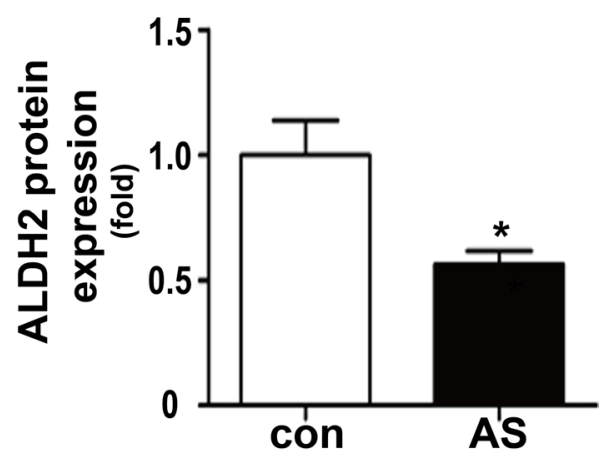

D

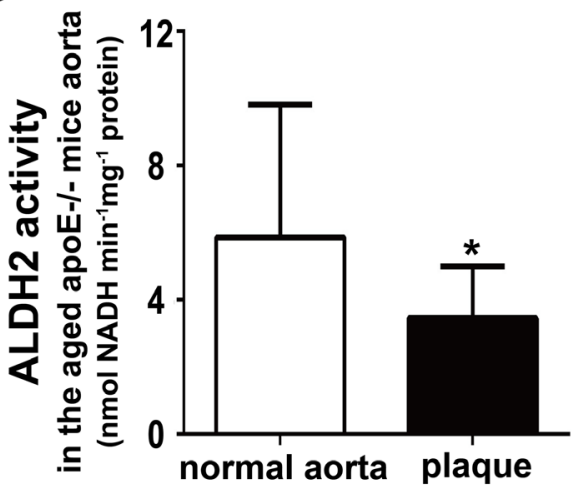

E
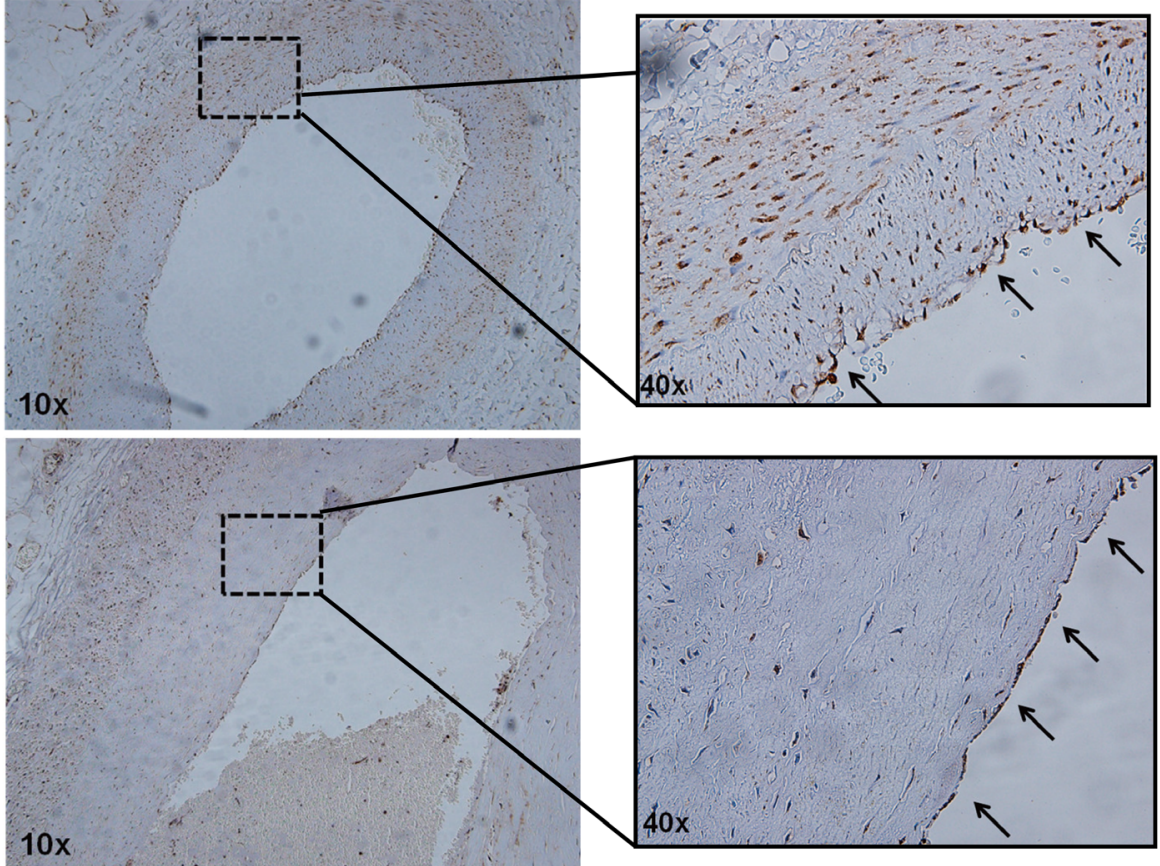

Figure 4: Expression and activity of ALDH2 in the vessels with or without atherosclerotic plaques. A. Representative blot of ALDH2 protein expression in human normal coronary arteries and atherosclerotic vessels. B. Statistical analysis of the data presented in Figure 4A. C. Histogram of ALDH2 activity in human normal coronary arteries and atherosclerotic vessels. D. Histogram of ALDH2 activity of old-aged apoE-/- mice aortas. E. Representative ALDH2 immunostaining in human coronary arteries. The arrows show that ALDH2 is expressed in endothelial cells. Con: normal coronary arteries, AS: atherosclerotic vessels. *: $P<0.05 v s$ control. The data were presented as mean \pm SEM. 
stability and inflammation through MAPK, NF- $\mathrm{B}$ and AP-1 signaling pathways, which suggests an important explanation for ALDH2 functioning as a CAD or ACS susceptibility gene and supplies solid evidence for ALDH2 effects on vasculature.

Previous pathological surveys in human have

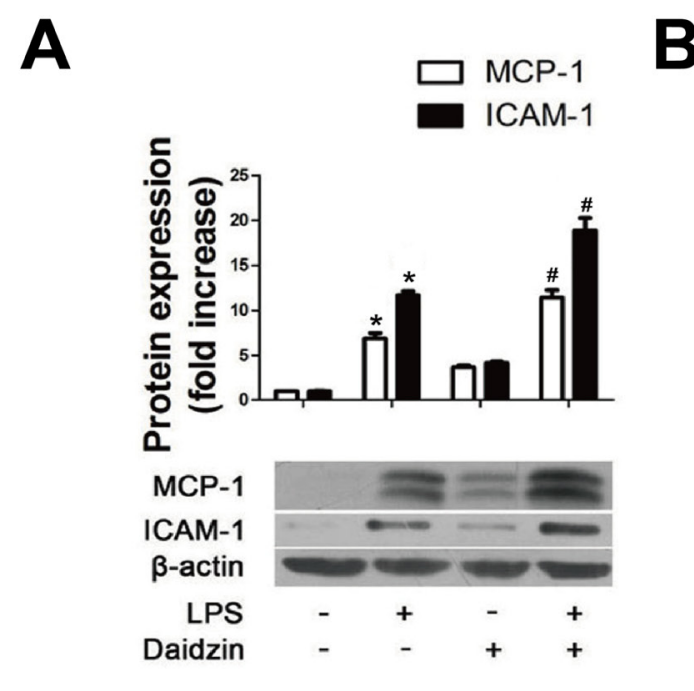

C

(CE)

(NE)

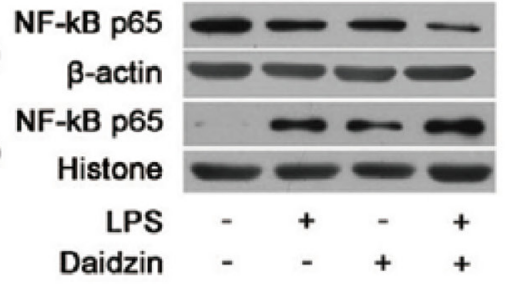

(CE)

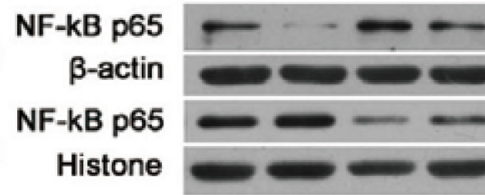

$\begin{array}{ccccc}\text { LPS } & - & + & - & + \\ \text { Alda-1 } & - & - & + & +\end{array}$
$\mathbf{B}$

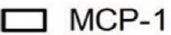

ICAM-1
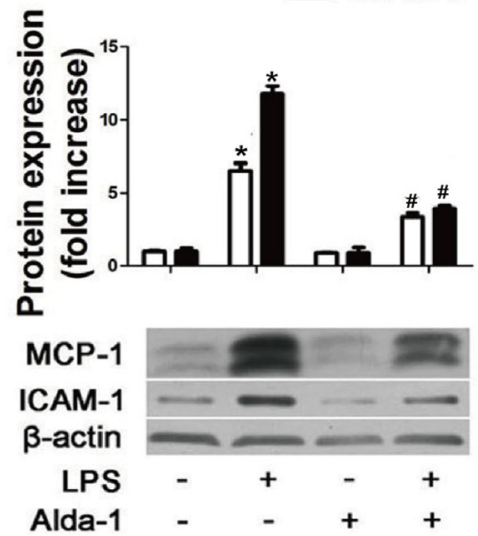

D

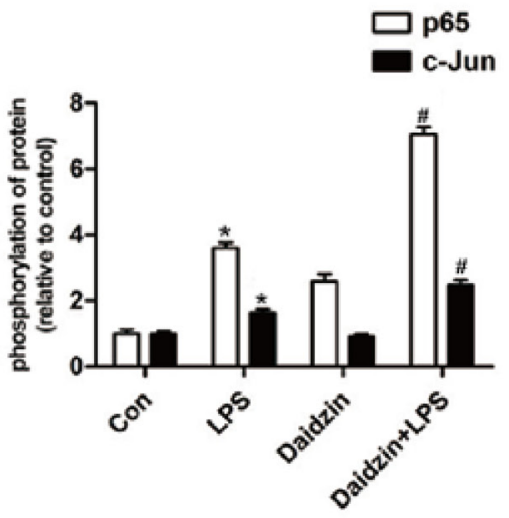

$\mathbf{F}$

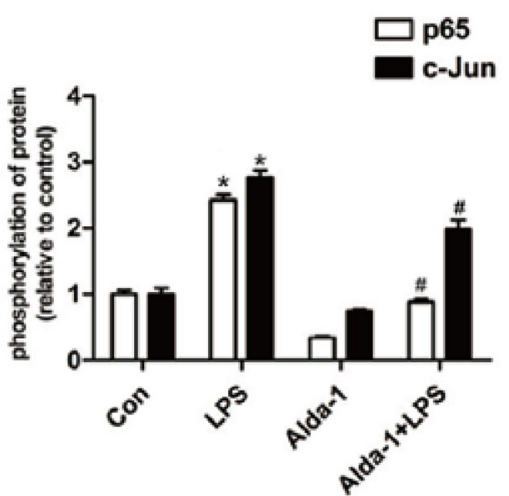

Figure 5: The effects of ALDH2 on MCP-1, ICAM-1, and NF- $\kappa B$ and AP-1 transcription factors in HUVECs. A. MCP-1 and ICAM-1 expression in HUVECs pre-treated with Daidzin before LPS treatment. B. MCP-1 and ICAM-1 expression in HUVECs pretreated with Alda-1 before LPS treatment. Values are expressed as means \pm SEM from three independent experiments. *: $P<0.05 v s$ control. \#: $P<0.05$ vs LPS treatment alone. C. NF-kB P65 protein expression in HUVECs pre-treated with or without Daidzin before LPS treatment. D. Phosphorylation of NF-kB P65 and AP-1 c-Jun in HUVECs with or without Daidzin before LPS treatment. E. Protein expression of NF-kB P65 in HUVECs treated with or without Alda-1 before LPS treatment. F. Phosphorylation levels of NF-kB P65 and AP-1 c-Jun in HUVECs with or without Alda- 1 before LPS treatment. Values are expressed as means \pm SEM from three independent experiments. *: $P<$ 0.05 vs control. " : $P<0.05$ vs LPS treatment alone. 

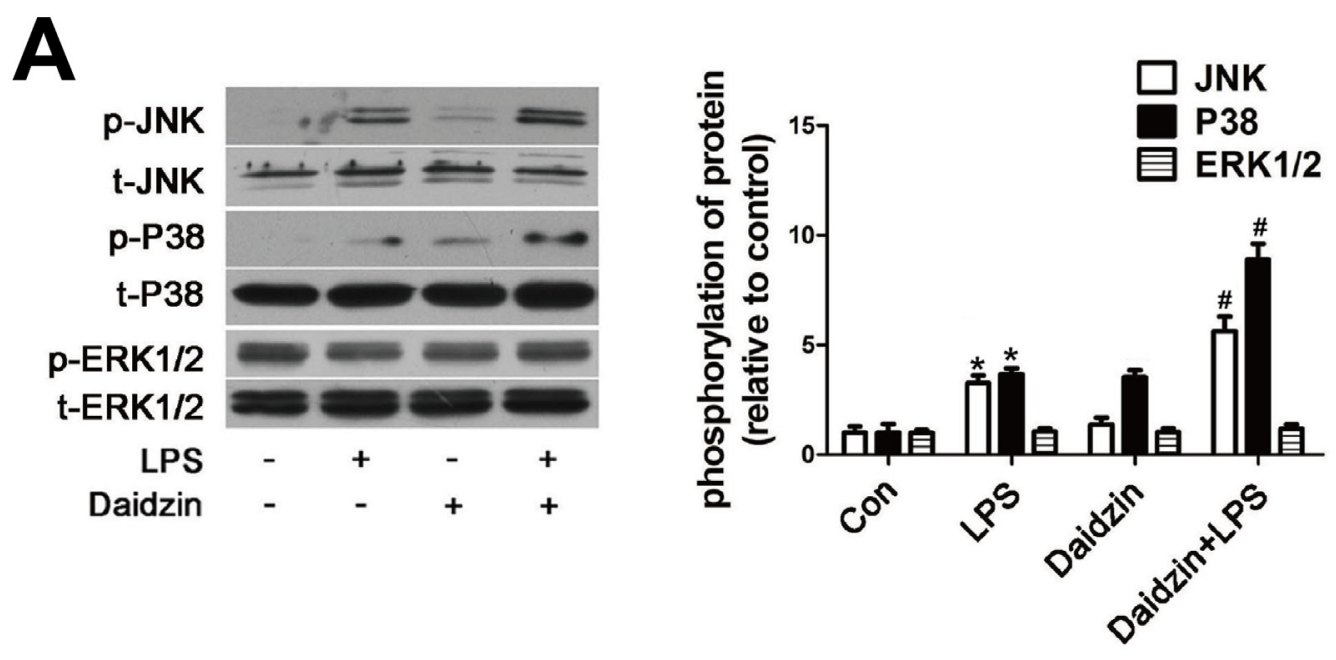

B
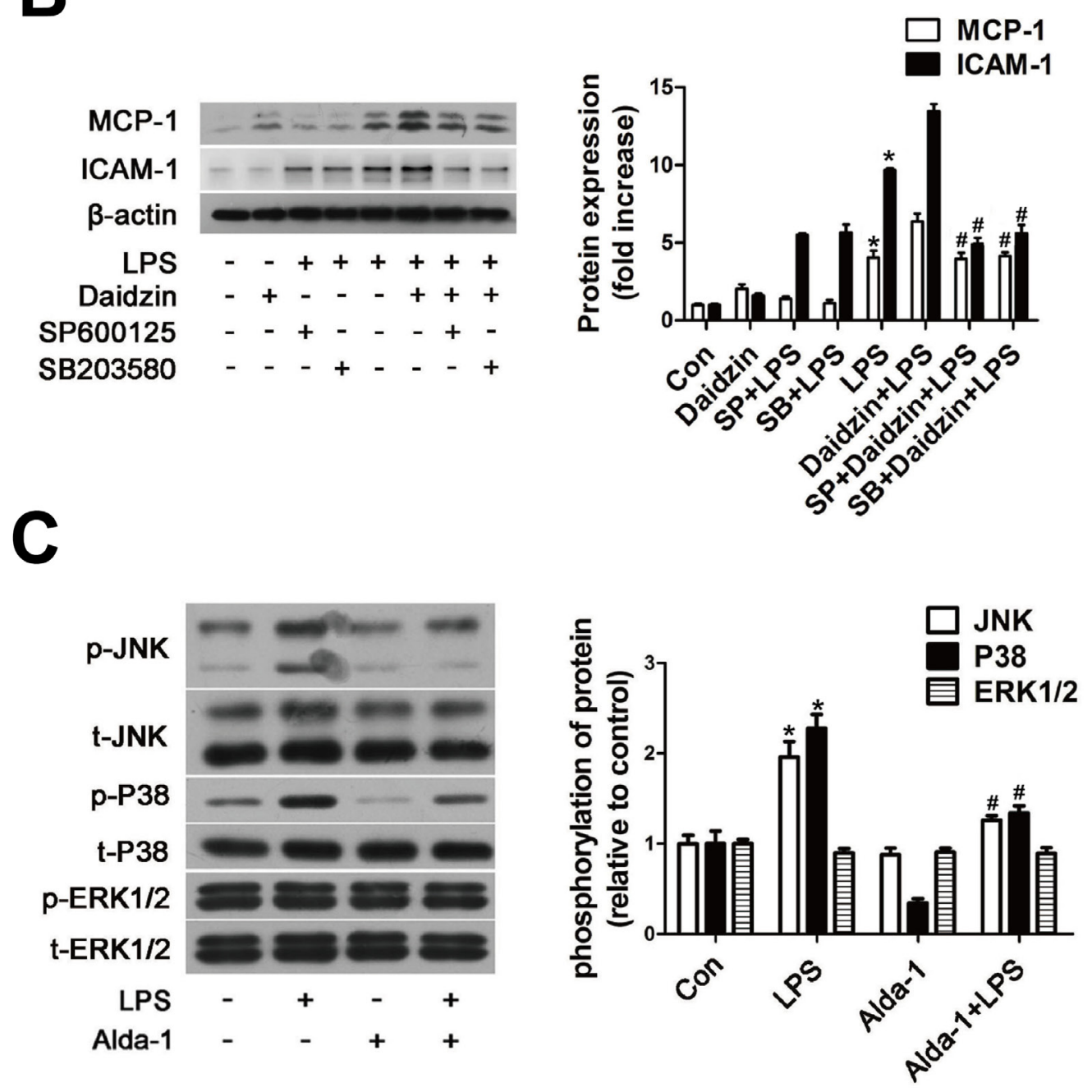

Figure 6: ALDH2 regulates expression of MCP-1 and ICAM-1 protein via JNK and P38 MAPK system in LPSactivated HUVECs. A. Phosphorylation of JNK, p38 MAPK and ERK1/2 in HUVECs stimulated by Daidzin before LPS treatment. *: $P<0.05 v s$ control. ": $P<0.05$ vs LPS treatment alone. B. MCP-1 and ICAM-1 expression after SP600125 $(20 \mu \mathrm{m})$ or SB203580 $(20 \mu \mathrm{m})$ treatment before LPS stimulation in HUVECs. Con: control; SP600125: JNK inhibitor; SB203580: P38 inhibitor. *: $P<0.05$ vs control. *: $P<0.05$ vs LPS+Daidzin treatment. C. Phosphorylation of JNK, p38 MAPK and ERK1/2 in HUVECs stimulated by Alda-1 before LPS treatment. *: $P<0.05$ vs control. " : $P<0.05$ vs LPS treatment alone. All values are expressed as means \pm SEM from three independent experiments. 
demonstrated that components of plaques rather than size of plaques play a more prominent role in the development of ACS, as its major cause is atherosclerotic plaque disruption, which may result from increased plaque vulnerability [29]. Plaque vulnerable to disruption is characterized by increased content of lipids and macrophages and reduced content of collagen and $\alpha$-SMCs [30]. To evaluate vulnerable plaques histologically, Shiomi put forward the "plaque vulnerability index", which incorporates the cumulative effects of the known morphological risk factors [31]. Our present data revealed that the Lv-ALDH2-overexpression group exhibited higher ALDH2 activity, reduced lesion area, less accumulation of lipids and macrophages, more collagen and SMCs in atherosclerotic plaques, with the Lv-ALDH2-RNAi exhibiting opposite effects. These findings suggest a key role for ALDH2 in governing components of atherosclerosis. Furthermore, this study demonstrated that all component changes of plaques in the Lv-ALDH2-overexpression group contributed to a decreased plaque vulnerability index, whereas those components of the Lv-ALDH2-RNAi group resulted in a much larger index. These results suggest that ALDH2 gene overexpression or ALDH2 with higher activity could enhance atherosclerotic plaque stability, whereas ALDH2 gene knockdown might promote plaque vulnerability. In addition, we found that there was no significant difference of HDL-C levels among the three groups, which seems that there was a discrepancy with previous human studies. In fact, according to previous studies, whether ALDH2 can influence serum lipids in human is still a controversial and uncertain issue, which should be still elucidated with studies in larger population sample in the future $[8,15,32$, 33]. Besides, the species difference between humans and mice may make a contribution to the discrepancy.

Existing evidence indicates that inflammation plays an important role in plaque instability. IL-6, MMP-2, ICAM-1 and MCP-1 are significant markers of inflammation and important inflammatory biomarkers to identify vulnerable plaques. In our present experiment, we observed that changes of expression of IL-6, MMP2, ICAM-1 and MCP-1 were negatively correlated with expression of ALDH2 protein. The protein levels of IL6, MMP-2, ICAM-1 and MCP-1 were reduced in the LvALDH2-overexpression group. Changes of these cytokines were simultaneous with a more stable plaque phenotype as manifested by increased collagen and $\alpha$-SMCs and decreased macrophages and lipids in the carotid plaques. However, the expression of these proteins was increased in the Lv-ALDH2-RNAi group, accompanying a less stable plaque. These results might suggest that ALDH2 may affect the components of plaques and plaque stability via inflammation.

In the present study, we also examined the relationship between $\mathrm{ALDH} 2$ and inflammation in
HUVECs treated with LPS in vitro. Our findings suggest that levels of two important inflammatory molecules, ICAM-1 and MCP-1, could be regulated by ALDH2, which was consistent with the in vivo results.

Among the known cell-signaling molecules mediating inflammation, $\mathrm{NF}-\kappa \mathrm{B}$ and $\mathrm{AP}-1$ are known to initiate the production of various inflammatory cytokines $[34,35]$. I $\mathrm{B}$ degradation through proteolysis and phosphorylation of NF- $\kappa \mathrm{B}$ p $65^{\mathrm{S} 536}$ contribute to the activity of $N F-\kappa B$ [28]. In this study, we observed that the NF- $\mathrm{B}$ p 65 levels in the nuclei of HUVECs and its phosphorylation induced by LPS were increased by ALDH2 inhibition but reduced by ALDH2 activation, consistent with the previous notion of NF- $\kappa \mathrm{B}$ in triggering inflammation. These results suggested that the effect of ALDH2 on the expression of ICAM-1 and MCP-1 was at least partly mediated by the regulation of $\mathrm{NF}$ $\kappa \mathrm{B}$ activity in HUVECs in response to LPS. Another nuclear transcription factor, AP-1, which has enhanced activity following phosphorylation, could bind to the upstream promoters of inflammatory cytokines to induce inflammation [36]. In this study, we observed that the phosphorylation level of AP-1 c-Jun induced by LPS was increased by ALDH2 inhibition and reduced by ALDH2 activation. These results clearly demonstrated that the mitochondrial ALDH2 significantly regulates NF- $\kappa$ B and AP-1 activity, and indicated that the effect of ALDH2 on the expression of ICAM-1 and MCP-1 was at least partly mediated by the regulation of $\mathrm{NF}-\kappa \mathrm{B}$ and $\mathrm{AP}-1$ activity in HUVECs in response to LPS.

MAPK signaling, including JNK, ERK1/2 and p38 MAPK, which can be activated by LPS and TNF- $\alpha$, is reported to play an important role in LPS-induced inflammation [37]. We assessed the effect of ALDH2 on LPS-induced phosphorylation of MAPKs. LPS-treated HUVECs exhibited increased phosphorylation levels of JNK and p38 MAPK, and both SP600125 and SB203580 reduced the expression of ICAM-1 and MCP-1. These results were consistent with previous studies [37]. These results revealed the critical roles of JNK and p38 MAPK in LPS-induced inflammation in HUVECs. Furthermore, our study demonstrated that phosphorylation of JNK and p38 MAPK was reduced by ALDH2 activation and enhanced by ALDH2 inhibition. Up to date, the detailed and precise mechanism underlying how ALDH2 inhibits JNK/p38$\mathrm{NF}-\kappa \mathrm{B}$ signaling pathway has not been fully established. This may be related to the clearance of toxic aldehydes by ALDH2 [38]. 4-HNE induces phosphorylation of JNK and activates it through down-regulation of HSP70, while ALDH2 overexpression detoxifies 4-HNE, leading to inhibited JNK phosphorylation. Moreover, acetaldehyde can induce p38 and JNK phosphorylation, while ALDH2 is the key enzyme for acetaldehyde detoxification [39, 40]. These JNK phosphorylation results are consistent with changes in the JNK downstream nuclear signaling AP-1 
phosphorylation following LPS treatment in HUVECs. Importantly, elevated ICAM-1 and MCP- 1 induced by ALDH2 inhibition in LPS-treated HUVECs were partly abolished by pretreatment of HUVECs with SP600125 and SB203580, suggesting that the JNK and p38 MAPK pathways are functionally involved in the regulation by ALDH2 of the expression of ICAM-1 and MCP-1 in LPSactivated HUVECs. On the other hand, the responses of SP600125 and SB203580 also revealed potential contributions of other signaling pathways in the effects of ALDH2 on inflammation.

Our data here for the first time provide convincing evidence that ALDH2 can influence atherosclerotic plaque development and vulnerability, and inflammatory response via the MAPK, NF- $\kappa \mathrm{B}$ and AP-1 signaling pathways. These results are potentially translational. Previous studies showed that continuous usage of some medications in CAD patients, such as nitrates, may drastically inhibit ALDH2 activity [41, 42]. Thus, clinical genotyping when necessary may offer an efficient way to promote early screening, personalized prevention and treatment, ultimately clinical guidance for this huge population, $\sim 6 \%$ of the world. These are particularly important during the era of personalized medicine. For susceptible population carrying ALDH2*504Lys allele, prevention and treatment of atherosclerotic diseases, such as CAD, may be optimized with cautious usage of certain medications including nitrates and the addition of drugs or life style modification (such as moderate drinking) to enhance ALDH2 activity while inhibiting inflammation [13, 41].

However, exactly how ALDH2 genetic profile in humans affects the clinical management of ACS remains elusive. Large scale multi-centered RCT studies are still warranted to understand the interplay between ALDH2 polymorphism and atherosclerosis. In addition, it is unrealistic to employee the histopathological techniques used in animal study for the detection of plaque formation in human subjects, making the clinical value of ALDH2 polymorphism more challenging for early diagnosis of atherosclerotic plaque formation using intravascular imaging techniques, such as intravascular ultrasound and optical coherence tomography. On the other hand, our limitation is that our findings from Lv-ALDH2-RNAi mice model can't directly reflect the function of ALDH2 rs671 mutant, therefore gene (wild-type and mutation) knock-in approach should be used to confirm our findings in the future studies.

\section{MATERIALS AND METHODS}

\section{Reagents and antibodies}

HUVEC medium was purchased from ScienCell (Carlsbad, CA). LPS, daidzin and oil-red O were purchased from Sigma (St. Louis, MO). Alda-1 was bought from Tocris Bioscience (USA). Sirius red was bought from Biohao biological technology (China). OptiMEM Medium and trypsin containing $0.25 \%$ EDTA were purchased from Gibco (Rockville, MD). Anti-ICAM-1 antibody and anti-ALDH2 antibody for western blot were obtained from Santa Cruz Biotechnology (Santa Cruz, CA). Anti-ALDH2 antibody for immunohistochemistry was bought from Proteintech (Chicago, USA). AntiMCP-1, anti-MOMA-2, anti-IL-6, anti-MMP-2 and antialpha-smooth muscle actin antibodies were purchased from Abcam (San Francisco, CA). Antibodies specific

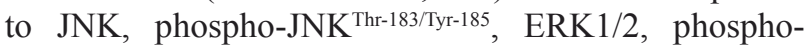
ERK1/2 $2^{\text {Thr-202/Tyr-204 }}$, P38 MAPK, phospho-P38 MAPK $^{\text {Thr-180/ }}$ Tyr-182, c-Jun, phospho-c-Jun ${ }^{\text {Ser-73 }}$ and $\beta$-actin were obtained from Cell Signaling Technology (Danvers, MA). Anti-

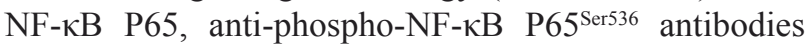
and SP600125 and SB203580 were purchased from the Beyotime Institute of Biotechnology (China). Secondary antibodies used for immunohistochemistry were bought from ZSGB biological technology (China). The constrictive collar (length-2.0 mm, inner diameter-0.30 $\mathrm{mm}$, outer diameter-0.50 $\mathrm{mm}$ ) was purchased from Shandong Key Laboratory of Medical Polymer Materials (China). The lentivirus vector ALDH2 construct was acquired from Genechem (China).

\section{Animal model}

Sixty male apoE-/- mice aged 8 weeks were purchased from the Beijing University Animal Research Center (Beijing, China). They were kept on a fixed 12 hour light/dark cycle at a constant temperature of $21.0 \pm 1.0^{\circ} \mathrm{C}$ with sufficient food and water. After anesthesia via an intraperitoneal injection of pentobarbital sodium $(50 \mathrm{mg} /$ $\mathrm{kg}$ ), a constrictive collar was placed around the right common carotid artery [43], and then the mice were fed a high-fat diet ( $15 \%$ cocoa butter and $0.25 \%$ cholesterol). Eight weeks later, the mice were divided into three groups: Lv-GFP group, Lv-ALDH2-overexpression group and LvALDH2-RNAi group $(\mathrm{n}=20$ each). Then, the lentiviral suspension of GFP, ALDH2 and ALDH2 siRNA was injected by cauda veins of the mice. Moreover, old-aged apoE-/- mice were used for ALDH2 activity detection. All animal procedures were in compliance with the NIH Guide and approved by the Animal Use and Care Committee of Shandong University. 


\section{Serum lipid levels}

At the end of the experiment, the mice were fasted overnight and blood samples were collected. The serum concentrations of total cholesterol, triglyceride, LDL-C and HDL-C were measured.

\section{Histopathological and immunohistochemical staining}

In all mice, the right common carotid arteries were collected for histopathological and immunohistochemical analysis. After being fixed with $4 \%$ formaldehyde (pH 7.2) overnight at $4{ }^{\circ} \mathrm{C}$, and embedded in OCT compound, the tissues samples from the right common carotid arteries were cross-sectioned into $7 \mu \mathrm{m}$ thick pieces at $50 \mu \mathrm{m}$ intervals. These sections were then stained with sirius red for collagen components and oil-red $\mathrm{O}$ for lipids, and immunohistochemical staining was used to analyze macrophages and $\alpha$-SMCs with specific antibodies. All sections were analyzed with an automated image analysis system, Image-Pro Plus 6.0 (Media Cybernetics, USA), for quantitative measurements. The vulnerability index was calculated according to the following formula: positive staining area of (macrophages + lipid) / positive staining area of ( $\alpha$-SMCs + collagen) [31]. Moreover, in order to quantify the extent of atherosclerotic lesions, the whole length of the aorta was excised for quantification of the en face plaque area. In brief, the aortas were opened longitudinally after carefully removing adventitial tissue, and stained with Oil red $\mathrm{O}$. The stereomicroscope was uesd to obtain the en face images, which were analyzed by ImageJ software. Percentage of the luminal surface area stained by Oil red-O was determined.

\section{Human coronary arteries samples preparation}

Coronary arteries from the dead donors were used to investigate which cells express ALDH2 and whether ALDH2 expression and activity changed in atherosclerotic vessels. Postmortem human coronary artery samples (n $=6$ ) with wild genotype were collected at autopsy from dead donors in Qilu Hospital, Shandong University. The study complies with the Declaration of Helsinki and was approved by the Institutional Ethics Committee at the institution. After formalin fixation and paraffin embedding, a part of human coronary arteries were serially sectioned at $5 \mu \mathrm{m}$ thickness, and these paraffin sections were used for ALDH2 immunohistochemical staining. And the remaining coronary arteries were frozen at $-80^{\circ} \mathrm{C}$ for western blotting and ALDH2 enzymatic activity detection.

\section{Cell culture}

HUVECs were purchased from the American type culture collection. For the experiments, 4-6 passages of the cells were applied and cultured in HUVEC medium containing $5 \%$ fetal bovine serum and $1 \%$ endothelial cell growth factors at $37^{\circ} \mathrm{C}$. When HUVECs reached $80 \%$ confluence, the complete medium was replaced by fresh HUVEC medium containing $0.05 \%$ fetal bovine serum. The ALDH2 inhibitor daidzin and ALDH2 activator Alda1 were applied 2 hours before LPS incubation.

\section{Extraction of mitochondria}

Mitochondria of mice aortas or HUVECs were prepared using a Tissue or Cell Mitochondrial isolation Kits (Beyotime, China) respectively. Briefly, the tissues or HUVECs were washed with $0.01 \mathrm{M}$ PBS, centrifuged and homogenized in mitochondrial isolation reagent. The resulting homogenate was centrifuged at $1,000 \times \mathrm{g}$ for $10 \mathrm{~min}$ and 3,500 $\times \mathrm{g}$ for $10 \mathrm{~min}$ at $4^{\circ} \mathrm{C}$. The resulting pellet contained the mitochondria. The mitochondria were disrupted, centrifuged at $12,000 \times \mathrm{g}$ for $10 \mathrm{~min}$ at $4^{\circ} \mathrm{C}$, and the supernatant was frozen at $-80^{\circ} \mathrm{C}$.

\section{Measurement of ALDH2 activity}

The activity of the mitochondrial ALDH2 was measured at room temperature in $50 \mathrm{mM}$ sodium pyrophosphate $(\mathrm{pH}=9.5)$ containing $2.5 \mathrm{mM} \mathrm{NAD}^{+}, 10$ $\mathrm{mM}$ acetaldehyde and $50 \mu \mathrm{g}$ of protein. Acetaldehyde as the substrate of ALDH2 was oxidized to acetic acid, whereas $\mathrm{NAD}^{+}$was reduced to NADH. Production of NADH was determined by spectrophotometric absorbance at $340 \mathrm{~nm}$. ALDH2 activity was expressed as nmol $\mathrm{NADH} / \mathrm{min} / \mathrm{mg}$ protein.

\section{Western blot analysis}

The mice carotid tissues, human coronary arteries or cell lysates of the same protein content (assayed by the BCA method; Bio-Rad, CA, USA) were prepared. Proteins were separated by $10 \%$ or $15 \%$ SDS-PAGE and transferred to nitrocellulose membranes. The membranes were blocked for 2 hours in a solution of $5 \%(\mathrm{wt} / \mathrm{vol}$.) milk and then incubated overnight at $4{ }^{\circ} \mathrm{C}$ with antibodies followed by horseradish peroxidase-conjugated rabbit anti-goat $(1: 5,000)$ or goat anti-rabbit $\operatorname{IgG}(1: 5,000$ or 1:10,000) for 2 hours. The bands were identified by a standard enhanced chemiluminescence method. ImageJ Software was used to quantitate the intensity of the bands. 


\section{Statistical analysis}

All the data were expressed as the mean \pm SEM. All data were assessed by student $t$ test or one-way ANOVA followed by Student-Newman-Keuls post-hoc analysis. P $<0.05$ was considered statistically significant.

\section{CONFLICTS OF INTEREST}

The authors declare no conflicts of interest.

\section{GRANT SUPPORT}

This work was supported by the National Natural Science Foundation of China (grant no. 81300103, 81300219, 81571934, 81522004); Taishan Scholar Program of Shandong Province (grant no. ts20130911); Specialized Research Fund for the Doctoral Program of Higher Education (grant no. 20130131110048); Key Technology Research and Development Program of Science and Technology of Shandong Province (grant no. 2014kjhm0102); and grants from Science and Technology of Shandong Province (grant no. ZR2013HQ012, 2014GSF118111); Science Foundation of Qilu Hospital of Shandong University and the Fundamental Research Funds of Shandong University (grant no. 2015QLMS37, 2014QLKY04, 2016QLQN02).

\section{Editorial note}

This paper has been accepted based in part on peerreview conducted by another journal and the authors' response and revisions as well as expedited peer-review in Oncotarget.

\section{REFERENCES}

1. Goedde HW, Agarwal DP, Harada S, Meier-Tackmann D, Ruofu D, Bienzle U, Kroeger A, Hussein L. Population genetic studies on aldehyde dehydrogenase isozyme deficiency and alcohol sensitivity. Am J Hum Genet. 1983;35:769-772.

2. Li Y, Zhang D, Jin W, Shao C, Yan P, Xu C, Sheng H, Liu Y, Yu J, Xie Y, Zhao Y, Lu D, Nebert DW, et al. Mitochondrial aldehyde dehydrogenase-2 (ALDH2) Glu504Lys polymorphism contributes to the variation in efficacy of sublingual nitroglycerin. The Journal of clinical investigation. 2006;116:506-511

3. Larson HN, Weiner H, Hurley TD. Disruption of the coenzyme binding site and dimer interface revealed in the crystal structure of mitochondrial aldehyde dehydrogenase “Asian" variant. J Biol Chem. 2005;280:30550-30556.

4. Li H, Borinskaya S, Yoshimura K, Kal'ina N, Marusin A, Stepanov VA, Qin Z, Khaliq S, Lee MY, Yang Y,
Mohyuddin A, Gurwitz D, Mehdi SQ, et al. Refined geographic distribution of the oriental ALDH2*504Lys (nee 487Lys) variant. Ann Hum Genet. 2009;73:335-345.

5. Zhang Y, Ren J. ALDH2 in alcoholic heart diseases: molecular mechanism and clinical implications. Pharmacol Ther. 2011;132:86-95.

6. Cichoz-Lach H, Partycka J, Nesina I, Celinski K, Slomka M, Wojcierowski J. Alcohol dehydrogenase and aldehyde dehydrogenase gene polymorphism in alcohol liver cirrhosis and alcohol chronic pancreatitis among Polish individuals. Scandinavian journal of gastroenterology. 2007;42:493498.

7. Jo SA, Kim EK, Park MH, Han C, Park HY, Jang Y, Song BJ, Jo I. A Glu487Lys polymorphism in the gene for mitochondrial aldehyde dehydrogenase 2 is associated with myocardial infarction in elderly Korean men. Clin Chim Acta. 2007;382:43-47.

8. Takagi S, Iwai N, Yamauchi R, Kojima S, Yasuno S, Baba T, Terashima M, Tsutsumi Y, Suzuki S, Morii I, Hanai S, Ono K, Baba S, et al. Aldehyde dehydrogenase 2 gene is a risk factor for myocardial infarction in Japanese men. Hypertens Res. 2002;25:677-681.

9. Kato N, Takeuchi F, Tabara Y, Kelly TN, Go MJ, Sim X, Tay WT, Chen CH, Zhang Y, Yamamoto K, Katsuya T, Yokota M, Kim YJ, et al. Meta-analysis of genome-wide association studies identifies common variants associated with blood pressure variation in east Asians. Nature genetics. 2011;43:531-538.

10. Takeuchi F, Yokota M, Yamamoto K, Nakashima E, Katsuya T, Asano H, Isono M, Nabika T, Sugiyama T, Fujioka A, Awata N, Ohnaka K, Nakatochi M, et al. Genome-wide association study of coronary artery disease in the Japanese. Eur J Hum Genet. 2012;20:333-340.

11. Hao PP, Xue L, Wang XL, Chen YG, Wang JL, Ji WQ, Xu F, Wei SJ, Zhang Y. Association between aldehyde dehydrogenase 2 genetic polymorphism and serum lipids or lipoproteins: a meta-analysis of seven East Asian populations. Atherosclerosis. 2010;212:213-216.

12. Wei SJ, Xing JH, Wang BL, Xue L, Wang JL, Li R, Qin WD, Wang J, Wang XP, Zhang MX, Chen YG. Poly(ADPribose) polymerase inhibition prevents reactive oxygen species induced inhibition of aldehyde dehydrogenase2 activity. Biochim Biophys Acta. 2013;1833:479-486.

13. Xue L, Xu F, Meng L, Wei S, Wang J, Hao P, Bian Y, Zhang Y, Chen Y. Acetylation-dependent regulation of mitochondrial ALDH2 activation by SIRT3 mediates acute ethanol-induced eNOS activation. FEBS Lett. 2012;586:137-142.

14. Wang J, Wang H, Hao P, Xue L, Wei S, Zhang Y, Chen Y. Inhibition of aldehyde dehydrogenase 2 by oxidative stress is associated with cardiac dysfunction in diabetic rats. Mol Med. 2011;17:172-179.

15. Xu F, Chen YG, Xue L, Li RJ, Zhang H, Bian Y, Zhang C, Lv RJ, Feng JB, Zhang Y. Role of aldehyde dehydrogenase 
2 Glu504lys polymorphism in acute coronary syndrome. J Cell Mol Med. 2011;15:1955-1962.

16. Ma H, Li J, Gao F, Ren J. Aldehyde dehydrogenase 2 ameliorates acute cardiac toxicity of ethanol: role of protein phosphatase and forkhead transcription factor. J Am Coll Cardiol. 2009;54:2187-2196.

17. Ma H, Guo R, Yu L, Zhang Y, Ren J. Aldehyde dehydrogenase 2 (ALDH2) rescues myocardial ischaemia/ reperfusion injury: role of autophagy paradox and toxic aldehyde. Eur Heart J. 2011;32:1025-1038.

18. Zhang H, Gong DX, Zhang YJ, Li SJ, Hu S. Effect of mitochondrial aldehyde dehydrogenase-2 genotype on cardioprotection in patients with congenital heart disease. Eur Heart J. 2012;33:1606-1614.

19. Wu B, Yu L, Wang Y, Wang H, Li C, Yin Y, Yang J, Wang Z, Zheng Q, Ma H. Aldehyde dehydrogenase 2 activation in aged heart improves the autophagy by reducing the carbonyl modification on SIRT1. Oncotarget. 2016;7:2175-2188. doi: 10.18632/oncotarget.6814.

20. Ray KK, Kastelein JJ, Boekholdt SM, Nicholls SJ, Khaw KT, Ballantyne CM, Catapano AL, Reiner Z, Luscher TF. The ACC/AHA 2013 guideline on the treatment of blood cholesterol to reduce atherosclerotic cardiovascular disease risk in adults: the good the bad and the uncertain: a comparison with ESC/EAS guidelines for the management of dyslipidaemias 2011. Eur Heart J. 2014;35:960-968.

21. Medzhitov R. Origin and physiological roles of inflammation. Nature. 2008;454:428-435.

22. Chen $\mathrm{CH}$, Budas GR, Churchill EN, Disatnik $\mathrm{MH}$, Hurley TD, Mochly-Rosen D. Activation of aldehyde dehydrogenase-2 reduces ischemic damage to the heart. Science. 2008;321:1493-1495.

23. Zhang H, Liu D, Wang X, Chen X, Long Y, Chai W, Zhou X, Rui X, Zhang Q, Wang H, Yang Q. Melatonin improved rat cardiac mitochondria and survival rate in septic heart injury. Journal of pineal research. 2013;55:1-6.

24. Keung WM, Vallee BL. Daidzin: a potent, selective inhibitor of human mitochondrial aldehyde dehydrogenase. Proc Natl Acad Sci U S A. 1993;90:1247-1251.

25. Perez-Miller S, Younus H, Vanam R, Chen CH, MochlyRosen D, Hurley TD. Alda-1 is an agonist and chemical chaperone for the common human aldehyde dehydrogenase 2 variant. Nat Struct Mol Biol. 2010;17:159-164.

26. Ding J, Zhang Q, Luo Q, Ying Y, Liu Y, Li Y, Wei W, Yan F, Zhang H. Alda-1 Attenuates Lung Ischemia-Reperfusion Injury by Reducing 4-Hydroxy-2-Nonenal in Alveolar Epithelial Cells. Critical care medicine. 2016.

27. Kumar A, Takada Y, Boriek AM, Aggarwal BB. Nuclear factor-kappaB: its role in health and disease. J Mol Med (Berl). 2004;82:434-448.

28. Sasaki CY, Barberi TJ, Ghosh P, Longo DL. Phosphorylation of RelA/p65 on serine 536 defines an I $\{$ kappa $\}$ B $\{$ alpha $\}$-independent NF- kappa $\}$ B pathway.
The Journal of biological chemistry. 2005;280:3453834547.

29. Steg PG, James SK, Atar D, Badano LP, BlomstromLundqvist C, Borger MA, Di Mario C, Dickstein K, Ducrocq G, Fernandez-Aviles F, Gershlick AH, Giannuzzi P, Halvorsen S, et al. ESC Guidelines for the management of acute myocardial infarction in patients presenting with ST-segment elevation. Eur Heart J. 2012;33:2569-2619.

30. Pundziute G, Schuijf JD, Jukema JW, Decramer I, Sarno G, Vanhoenacker PK, Boersma E, Reiber JH, Schalij MJ, Wijns W, Bax JJ. Evaluation of plaque characteristics in acute coronary syndromes: non-invasive assessment with multi-slice computed tomography and invasive evaluation with intravascular ultrasound radiofrequency data analysis. Eur Heart J. 2008;29:2373-2381.

31. Shiomi M, Ito T, Hirouchi Y, Enomoto M. Fibromuscular cap composition is important for the stability of established atherosclerotic plaques in mature WHHL rabbits treated with statins. Atherosclerosis. 2001;157:75-84.

32. Xu F, Sun Y, Shang R, Li M, Cui L, Cui Z, Chen Y. The Glu504Lys polymorphism of aldehyde dehydrogenase 2 contributes to development of coronary artery disease. The Tohoku journal of experimental medicine. 2014;234:143150.

33. Hashimoto Y, Nakayama T, Futamura A, Omura M, Nakarai H, Nakahara K. Relationship between genetic polymorphisms of alcohol-metabolizing enzymes and changes in risk factors for coronary heart disease associated with alcohol consumption. Clin Chem. 2002;48:1043-1048.

34. Abdel-Malak NA, Srikant CB, Kristof AS, Magder SA, Di Battista JA, Hussain SN. Angiopoietin-1 promotes endothelial cell proliferation and migration through AP1-dependent autocrine production of interleukin-8. Blood. 2008;111:4145-4154.

35. Isoda K, Young JL, Zirlik A, MacFarlane LA, Tsuboi N, Gerdes N, Schonbeck U, Libby P. Metformin inhibits proinflammatory responses and nuclear factor-kappaB in human vascular wall cells. Arteriosclerosis, thrombosis, and vascular biology. 2006;26:611-617.

36. Allison CC, Kufer TA, Kremmer E, Kaparakis M, Ferrero RL. Helicobacter pylori induces MAPK phosphorylation and AP-1 activation via a NOD1-dependent mechanism. J Immunol. 2009;183:8099-8109.

37. Wei L, Matsumoto H, Yamaguchi H. Propofol attenuates lipopolysaccharide-induced monocyte chemoattractant protein-1 production through $\mathrm{p} 38 \mathrm{MAPK}$ and SAPK/JNK in alveolar epithelial cells. Journal of anesthesia. 2013;27:366373.

38. Sun A, Zou Y, Wang P, Xu D, Gong H, Wang S, Qin Y, Zhang P, Chen Y, Harada M, Isse T, Kawamoto T, Fan $\mathrm{H}$, et al. Mitochondrial aldehyde dehydrogenase 2 plays protective roles in heart failure after myocardial infarction via suppression of the cytosolic JNK/p53 pathway in mice. J Am Heart Assoc. 2014;3:e00779. 
39. Li SY, Li Q, Shen JJ, Dong F, Sigmon VK, Liu Y, Ren J. Attenuation of acetaldehyde-induced cell injury by overexpression of aldehyde dehydrogenase-2 (ALDH2) transgene in human cardiac myocytes: role of MAP kinase signaling. J Mol Cell Cardiol. 2006;40:283-294.

40. Li SY, Gomelsky M, Duan J, Zhang Z, Gomelsky L, Zhang X, Epstein PN, Ren J. Overexpression of aldehyde dehydrogenase-2 (ALDH2) transgene prevents acetaldehyde-induced cell injury in human umbilical vein endothelial cells: role of ERK and p38 mitogen-activated protein kinase. J Biol Chem. 2004;279:11244-11252.

41. Murphy TC, Arntzen R, Picklo MJ, Sr. Nitratebased vasodilators inhibit multiple vascular aldehyde dehydrogenases. Cardiovasc Toxicol. 2005;5:321-332.
42. Sydow K, Daiber A, Oelze M, Chen Z, August M, Wendt M, Ullrich V, Mulsch A, Schulz E, Keaney JF, Jr., Stamler JS, Munzel T. Central role of mitochondrial aldehyde dehydrogenase and reactive oxygen species in nitroglycerin tolerance and cross-tolerance. J Clin Invest. 2004;113:482489.

43. von der Thusen JH, van Berkel TJ, Biessen EA. Induction of rapid atherogenesis by perivascular carotid collar placement in apolipoprotein E-deficient and low-density lipoprotein receptor-deficient mice. Circulation. 2001;103:1164-1170. 